\title{
Influence of Operational Variables on Ball-Milling of Sulfadimethoxine and White Alundum
}

\author{
Nobuyoshi Kaneniwa, Akiko Ikekawa, and Katsuyo Hashimoto \\ School of Pharmacentical Sciences, Showa University ${ }^{1)}$
}

(Received November 11, 1972)

In the previous paper, thirty two kinds of organic and inorganic powders were ball-milled and the rate of an increase of surface area by ball-milling was considered to depend on coherency of powder particles expressed as a function of surface energy, melting point, solubility in water, true density and so on.2) In this paper, influence of operational variables on the rate of an increase of surface area of sulfadimethoxine and white alundum by ball-milling was investigated.

\section{Experimental}

The materials used were sulfadimethoxine offered from Chugai Seiyaku Kogyo Co. and white alundum (Type 500) purchased from Nishio Kogyo Co.

Various amounts of ceramic balls with true density of $2.4 \mathrm{~g} / \mathrm{cm}^{3}$ and in diameter between $1.4 \mathrm{~cm}$ and $3.5 \mathrm{~cm}$ were inserted in a ceramic mill in the inside diameter between $8 \mathrm{~cm}$ and $14 \mathrm{~cm}$ and in capacity between $450 \mathrm{ml}$ and $3430 \mathrm{ml}$. Various amounts of stainless steel balls with true density of $8.2 \mathrm{~g} / \mathrm{cm}^{3}$ and in diameter of $1.9 \mathrm{~cm}$ and $2.5 \mathrm{~cm}$ were inserted in a stainless steel mill in the inside diameter of $10 \mathrm{~cm}$ and in capacity of $870 \mathrm{ml}$. Various maounts of materials were ball-milled in these mills with the revolving velocity of a mill between $90 \mathrm{rpm}$ and $210 \mathrm{rpm}$. Surface area of ball-milled samples was measured by air permeability method. ${ }^{3}$ )

\section{Result and Discussion}

The rate of an increase of surface area decreased gradually with the lapse of ball-milling time. (Fig. 1) As shown in Fig. 2, equation (1) applied well, where $\mathrm{S}$ was specific surface area of a sample, and $k_{1}$ and $k_{2}$ were parameters dependent on physicochemical properties of ball-milled samples, operational variables and so on. ${ }^{2)}$

$$
d S / d t=k_{1} \exp \left(-k_{2} S\right)
$$

Parameter $k_{1}$ is identical with $d S / d t$ for the sample whose surface area is negligibly small. In the previous paper, $k_{2}$ was small for the sample with high melting point and little solubility in water. ${ }^{2)}$ Probably, $k_{2}$ is a parameter concerning a decrease of $d S / d t$ due to coherency of particles caused by an increase of surface area by ball-milling.

\section{1) Influence of a Revolving Velocity of a Mill on Parameters $\boldsymbol{k}_{\mathbf{1}}$ and $\boldsymbol{k}_{2}$}

The way how balls move in a revolving mill is considered to depend on the balance of the gravitational force of balls and the centrifugal force given to balls by the revolution of a mill. If $N_{\mathrm{c}}$ is the revolving velocity of a mill when the gravitational force of balls is equal to the centrifugal force, equation (2) is applied and $N_{\mathrm{c}}$ is represented by equation (3), where $m_{\mathrm{b}}$ is the weight of a ball, $D$ is the inside diameter of a mill, $d$ is a diameter of a ball and $g$ is the gravitational constant.

1) Hatanodai, 1-5-8, Shinagawa-ku, Tokyo.

2) A. Ikekawa, K. Imagawa, T. Omori, N. Kaneniwa, Chem. Pharm. Bull. (Tokyo), 19, 1027 (1971).

3) E. Suito, M. Arakawa, M. Takahashi, Kogyo Kagaku Zasshi, 59, 307 (1956). 


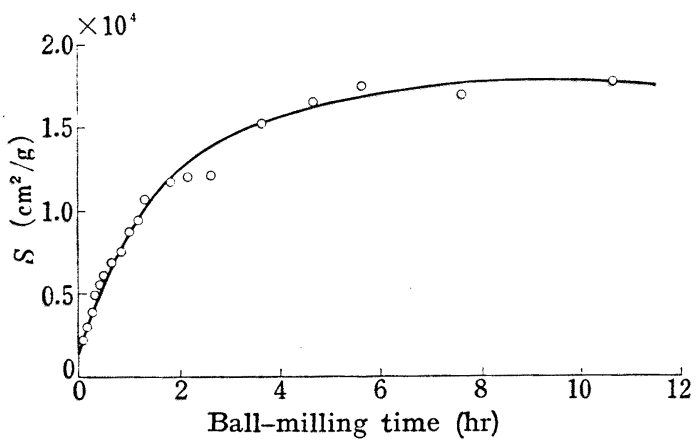

Fig. 1. Increase of Surface Area of Sulfadimethoxine with the Lapse of Ball-Milling Time

ceramic mill, $D=8 \mathrm{~cm}, N / N_{\mathrm{c}}=0.76, W_{\mathrm{s}}=20 \mathrm{~g}, J_{\mathrm{b}}=0.2 \mathrm{e}$

The ratio of the number of balls in diameter of $2.5 \mathrm{~cm}$ to that of balls in diameter of $1.9 \mathrm{~cm}$ was $1 / 3$.

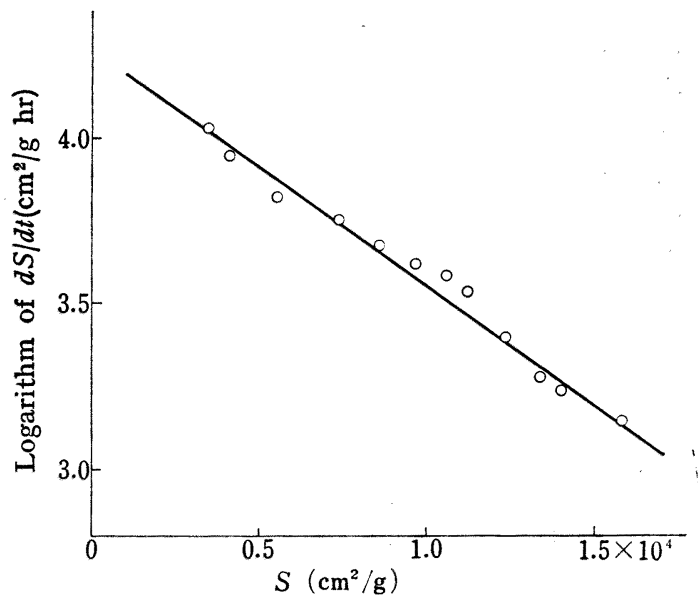

Fig. 2. Application of Equation (1) to Ball-Milling of Sulfadimethoxine

ceramic mill, $D=8 \mathrm{~cm}, N / N_{\mathrm{c}}=0.76, W_{\mathrm{g}}=20 \mathrm{~g}, J_{\mathrm{b}}=0.25$

The ratio of the number of balls in diameter of $2.5 \mathrm{~cm}$ to that of balls in diameter of $1.9 \mathrm{~cm}$ was $1 / 3$.

$$
\begin{aligned}
& m_{\mathrm{b}} g=(1 / 2) m_{\mathrm{b}}(D-d)\left(2 \pi N_{\mathrm{c}}\right)^{2} \\
& N_{\mathrm{c}}=(1 / 2 \pi) \sqrt{2 g /(D-d)}
\end{aligned}
$$

A transparent acrylic lid was sticked on a mill with a vinyl tape and the movement of balls in a revolving mill was observed. When the revolving velocity of a mill, $N$, was smaller than $N_{\mathrm{c}}$, balls rolled down over the layers of another balls successively after held up to a certain height by the wall of a revolving mill. Matsui, et al. reported that a decrease of particle size was mainly attributed to impact stress of balls rolling down over the layers of another balls when the apparent volume of balls in loosest packing was nearly half the volume of a mill, the apparent volume of a sample was approximately equal to the space between balls in loosest packing and $N / N_{\mathrm{c}}$ was $0 . .^{4}$ ) When $N / N_{\mathrm{c}}$ was larger than 1.0 , the balls rotating along the wall of a mill by a centrifugal force collided each another. In this case, a decrease of particle size is probably attributed to impact stress by collision between balls.

Porosity of balls, $\varepsilon_{\mathrm{b}}$, was assumed to be 0.50 in consideration of a wall effect because of the large value of $d / D$, and the ratio of the apparent volume of balls in loosest packing to the inside volume of a mill, $J_{\mathrm{b}}$, was calluculated by equation (4), where $W_{\mathrm{b}}$ was the total weight of balls, $\rho_{\mathrm{b}}$ was the true density of balls and $V_{\mathrm{m}}$ was the inside volume of a mill.

$$
J_{\mathrm{b}}=\left(W_{\mathrm{b}} / \rho_{\mathrm{b}}\right) / V_{\mathrm{m}}\left(1-\varepsilon_{\mathrm{b}}\right)
$$

In case of 0.42 of $J_{\mathrm{b}}, k_{1} / N$ was the largest and $k_{2}$ was the smallest when $N / N_{\mathrm{c}}$ was around 0.70 . When $J_{\mathrm{b}}$ was equal to or larger than 0.65 , both $k_{1} / N$ and $k_{2}$ decreased with an increase of $N / N_{\mathrm{c}}$. (Fig. 3 and 4) When $J_{\mathrm{b}}$ was 0.65 , the distance between the lid of a mill and the top of the layers of balls was approximately equal to the diameter of balls because of the large value of $d / D$, and it was a little difficult to obtain $J_{\mathrm{b}}$ larger than 0.65 . Balls may move more smoothly as $N / N_{\mathrm{c}}$ decreased, in case of $J_{\mathrm{b}}$ equal to or larger than 0.65 . In this case, a decrease of particle size is probably due to compression and shear between balls or between balls and the wall of a mill. 


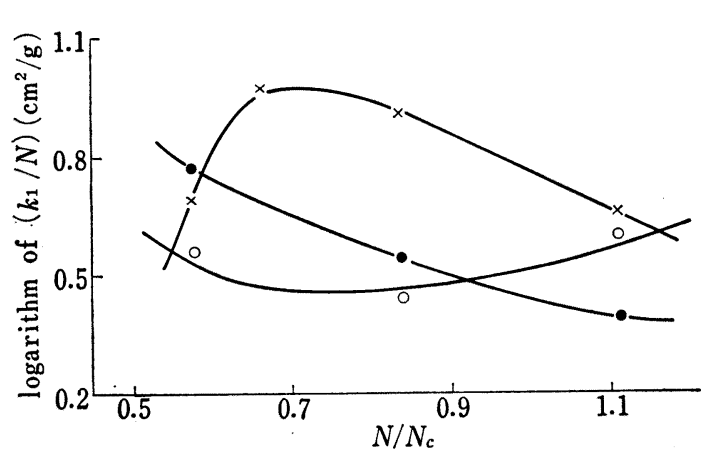

Fig. 3. Influence of the Revolving Velocity of a Mill on Parameter $k_{1}$ for Sulfadimethoxine

ceramic mill, $D=8 \mathrm{~cm}, W_{\mathrm{s}}=30 \mathrm{~g}$,

The ratio of the number of balls in diameter of $2.5 \mathrm{~cm}$ to that of balls in diameter of $1.9 \mathrm{~cm}$ was $1 / 3$.

$J_{\mathrm{b}}: \bigcirc: 017, x: 0.42,0: 0.65$

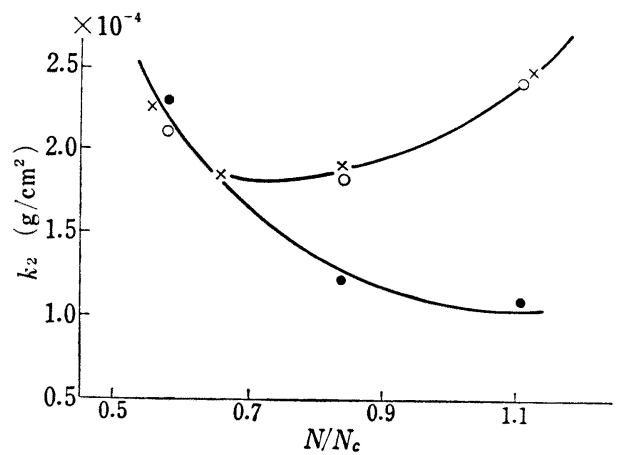

Fig. 4. Influence of the Revolving Velocity of a Mill on Parameter $k_{2}$ for Sulfadimethoxine

ceramic mill, $D=8 \mathrm{~cm}, W_{\mathrm{B}}=30 \mathrm{~g}$

The ratio of the number of balls in diameter of $2.5 \mathrm{~cm}$ to that of the balls in diameter of $1.9 \mathrm{~cm}$ was $1 / 3$.

$J_{\mathrm{b}}: \bigcirc: 0.17, x: 0.42,0: 0.65$

\section{2) Influence of $\boldsymbol{J}_{\mathrm{b}}$ on Parameters $\boldsymbol{k}_{\mathbf{1}}$ and $\boldsymbol{k}_{\mathbf{2}}$}

In case of $N / N_{\mathrm{c}}$ smaller than 1.0, $k_{1}$ increased in proportion to the $\alpha$ th power of $J_{\mathrm{b}}$ when $J_{\mathrm{b}}$ was smaller than 0.5 , and $\alpha$ was 1.8 for sulfadimethoxine in case of 0.85 of $N / N_{\mathrm{c}}$ and $\alpha$ was 2.1 for sulfadimethoxine in case of 0.55 of $N / N_{\mathrm{c}}$ and for white alundum in case of 0.70 of $N / N_{\mathrm{c}}$. When $J_{\mathrm{b}}$ was larger than $0.50, k_{1}$ decreased with an increase of $J_{\mathrm{b}}$. But $k_{1}$ increased again when $J_{\mathrm{b}}$ was equal to or larger than 0.65 and $N / N_{\mathrm{c}}$ was smaller than 0.85 . (Fig. 5) In case of $N / N_{\mathrm{c}}$ larger than $1.0, k_{1}$ was large and influenced little by $J_{\mathrm{b}}$ between 0.15 and 0.45 . (Fig. 6) Parameter $k_{2}$ was influenced little by $J_{\mathrm{b}}$.

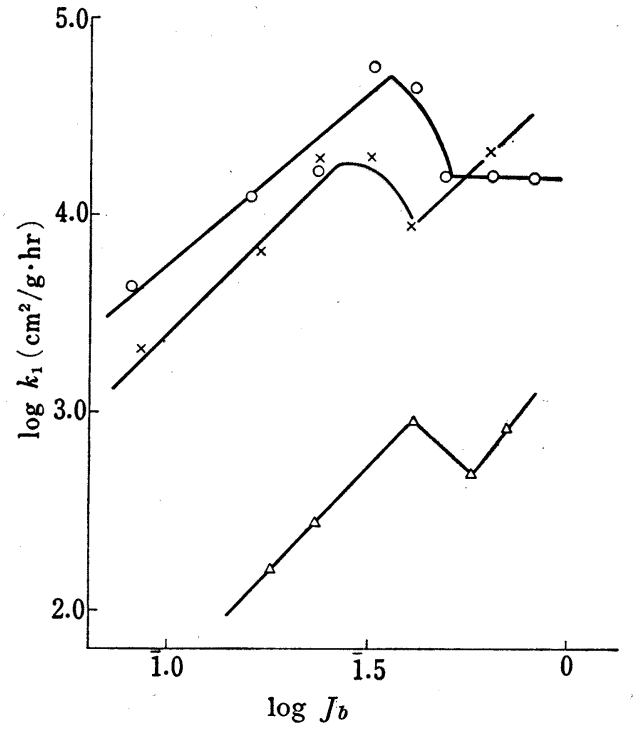

Fig. 5. Influence of $J_{\mathrm{b}}$ on Parameter $k_{1}$ for Sulfadimethoxine and White Alundum

$$
\begin{aligned}
& \text { ceramic mill, } \mathrm{D}=8 \mathrm{~cm} \\
& \text { sulfadimethoxine, } W_{\mathrm{s}}=20 \mathrm{~g}, N / N_{\mathrm{C}}=0.85, \bigcirc \\
& W_{\mathrm{s}}=30 \mathrm{~g}, N / N_{\mathrm{C}}=0.55 \times \\
& \text { The ratio of the number of balls in diameter of } \\
& 2.5 \mathrm{~cm} \text { to that of balls in diameter of } 1.9 \mathrm{~cm} \text { was } \\
& 1 / 3 .
\end{aligned}
$$

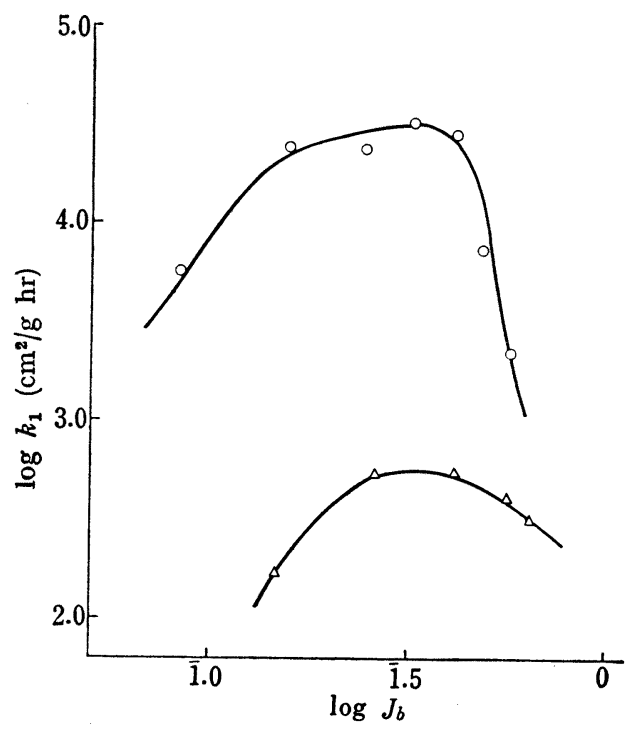

Fig. 6. Influence of $J_{\mathrm{b}}$ on Parameter $k_{1}$ for Sulfadimethoxine and White Alundum

$$
\begin{aligned}
& \text { ceramic mill, } D=8 \mathrm{~cm}, N / N_{\mathrm{C}}=1.1 \\
& : \text { :sulfadimethoxine, } W_{\mathrm{S}}=20 \mathrm{~g} \\
& \text { The ratio of the number of balls in diameter of } 2.5 \\
& \text { cm to that of balls in diameter of } 1.9 \mathrm{~cm} \text { was } 1 / 3 . \\
& \Delta: \text { white alundum, } W_{\mathrm{S}}=25 \mathrm{~g}, d=1.9 \mathrm{~cm}
\end{aligned}
$$




\section{3) Influence of the Apparent Volume of the Inserted Sample on Parameters $\boldsymbol{k}_{1}$ and $\boldsymbol{k}_{2}$}

Approximately, $k_{1}$ was inversely proportional to the 1.6 th power of the volume of the inserted sample in case of 0.85 of $N / N_{\mathrm{c}}$ in the previous paper. ${ }^{2)}$ But detailed investigation showed that $k_{1}$ for the case that the apparent volume of the sample was much larger than the space between balls in loosest packing was smaller than the value expected from the straight line obtained by the logarithmic plot of $k_{1}$ versus the volume of the sample equal to or smaller than the space between balls in loosest packing. The ratio of the apparent volume of the sample to the inside volume of a mill was represented by equation (5), where $W_{\mathrm{s}}$ was the total weight of the sample, $\rho_{\mathrm{s}}$ was the true density of the sample and $\varepsilon_{\mathrm{s}}$ was the porosity of the sample.

$$
J_{\mathrm{s}}=\left(W_{\mathrm{s}} / \rho_{\mathrm{s}}\right) / V_{\mathrm{m}}\left(1-\varepsilon_{\mathrm{s}}\right)
$$

In the previous paper, porosity of crushed white alundum with specific surface diameter between $1.0 \mu$ and $10.0 \mu$ in loosest packing was between 0.75 and 0.80 , and that of the crushed sulfadimethoxine with specific diameter between $1.0 \mu$ and $30.0 \mu$ in loosest packing was between 0.70 and $0.75 .^{2)}$ Then, the values of $J_{\mathrm{s}}$ were calcullated by equation (5), under the assumption that $\varepsilon_{\mathrm{b}}$ for white alundum was 0.75 and that for sulfadimethoxine was 0.70 . The value of $J_{\mathrm{s}} / \varepsilon_{\mathrm{b}} J_{\mathrm{b}}$ is identical with the ratio of the apparent volume of the sample to the space between balls in loosest packing. As shown in Fig. 7, straight lines were obtained by the logarithmic plot of $k_{1}$ versus $J_{\mathrm{s}} / \varepsilon_{\mathrm{b}} J_{\mathrm{b}}$ smaller than 1.0 , and $k_{1}$ in case of $J_{\mathrm{s}} / \varepsilon_{\mathrm{b}} J_{\mathrm{b}}$ larger than 1.0 was under these lines. The tangent of these straight lines, $-\beta$, was small for sticky powders and in case of large $N / N_{\mathrm{c}}$ (Table I). Parameter $k_{2}$ was influenced little by the apparent volume of the sample inserted in a mill.

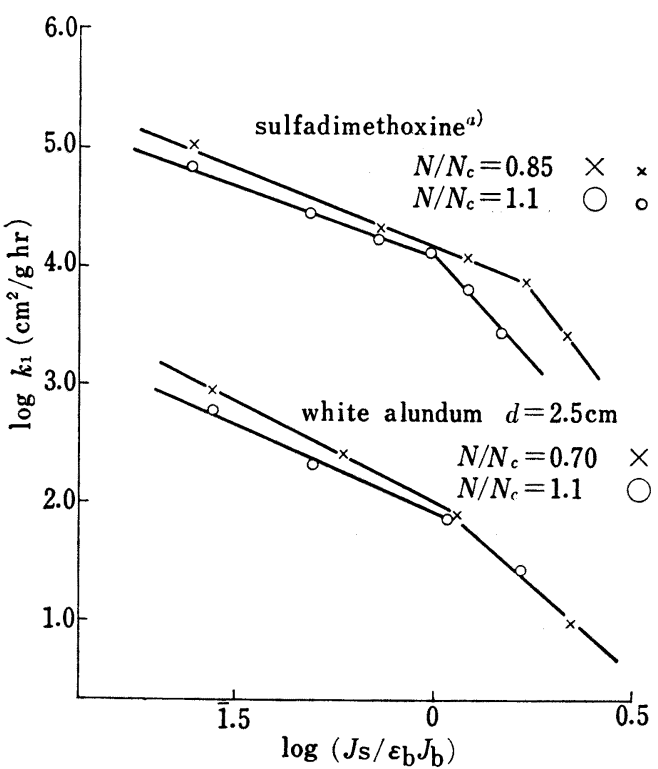

Fig. 7. Influence of the Apparent Volume of Sulfadimethoxine and White Alundum Inserted in a Mill on Parameter $k_{1}$

ceramic mill, $D=8 \mathrm{~cm}, J_{\mathrm{b}}=0.42$

a) In case of sulfadimethoxine, the ratio of the number of balls in diameter of $2.5 \mathrm{~cm}$ to that of balls in diameter of $1.9 \mathrm{~cm}$ was $1 / 3$.
TABLE I. The Value of $\beta$ for Sulfadimethoxine and White Alundum

\begin{tabular}{lccc}
\hline & \multicolumn{3}{l}{$\begin{array}{l}\text { Ceramic mill, } D=8 \\
\mathrm{~cm}, J_{\mathrm{b}}=0.42\end{array}$} \\
\cline { 3 - 4 } & \multicolumn{2}{c}{$N / N_{\mathrm{c}}$} & $\beta$ \\
\hline Sulfadimethoxine & $a)$ & 0.85 & 1.36 \\
& $a)$ & 1.1 & 1.14 \\
White alundum & $2.5 \mathrm{~cm}$ & 0.70 & 1.72 \\
& $2.5 \mathrm{~cm}$ & 1.1 & 1.48 \\
\hline
\end{tabular}

a) The ratio of the number of balls in diameter of $2.5 \mathrm{~cm}$ to that of balls in diameter of $1.9 \mathrm{~cm}$ was $1 / 3$.

4) Influence of the Inside Diameter of a Mill and the Diameter of Balls on Parameters $k_{1}$ and $k_{2}$

As shown in Fig. 8 and 9 , the inside diameter of a mill and the diameter of balls influenced little on parameters $k_{1}$ and $k_{2}$. The same results were also obtained by ballmilling eleven kinds of organic and inorganic powders other than sulfadimethoxine and white alundum. Fig. 9 shows the result for ball-milling by balls of an equal size. The

value of $k_{1}$ for ball-milling by the mixture of large balls and small balls was identical with the value for ball-milling by balls of an equal size. 


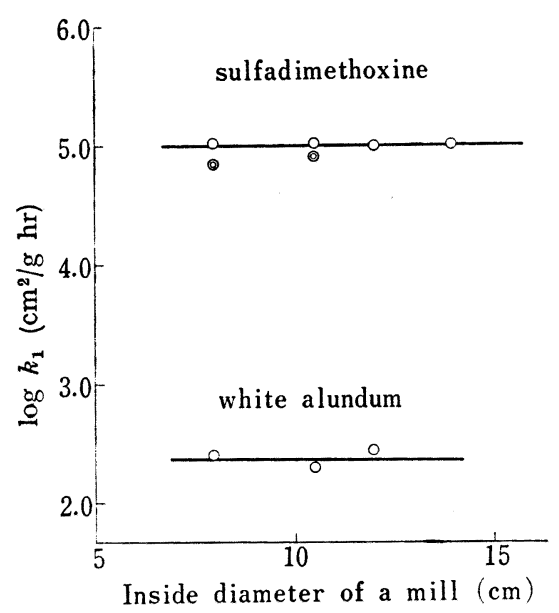

Fig. 8. Influence of the Inside Diameter of a Mill on Parameter $k_{1}$ for Sulfadimethoxine and White Alundum

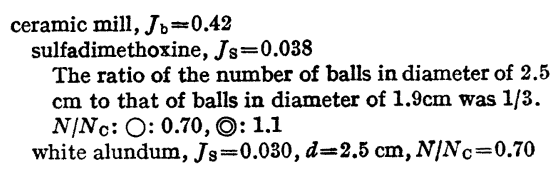

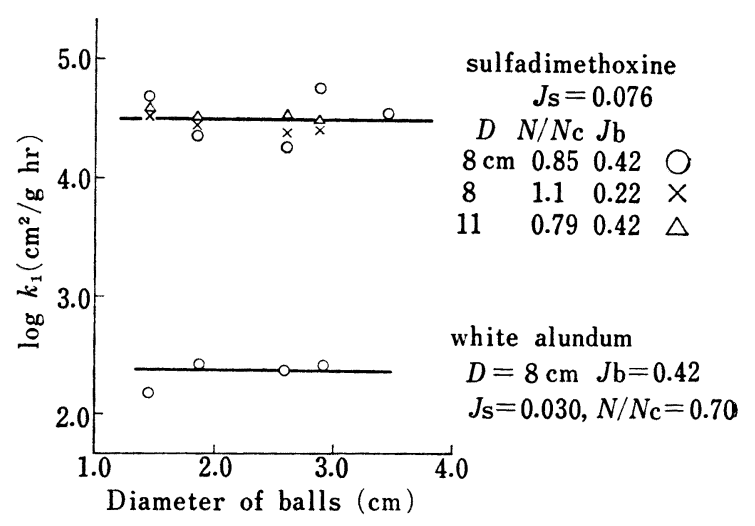

Fig. 9. Influence of the Diameter of the Balls on Parameter $k_{1}$ for Sulfadimethoxine and White Alundum (Ceramic mill)

\section{5) Influence of the True Density of Balls on Parameters $\boldsymbol{k}_{1}$ and $\boldsymbol{k}_{2}$}

The value of $k_{1}$ for ball-milling by stainless steel balls was larger than the value for ballmilling by ceramic balls. If $k_{1}$ was proportional to the $\gamma$ th power of the true density of balls, $\gamma$ was approximately 1.0 in case of $N / N_{\mathrm{c}}$ smaller than 1.0 and a little smaller than 1.0 in case of $N / N_{\mathrm{c}}$ larger than 1.0. (Table II). Parameter $k_{2}$ was influenced little by the true density of balls.

TABLE II. Influence of the True Density of Balls on Parameter $k_{1}$ for Sulfadimethoxine and White Alundum

Stainless steel mill: $D=10 \mathrm{~cm}$, Ceramic mill: $D=11 \mathrm{~cm} J \mathrm{~s}=0.076, J_{\mathrm{b}}=0.42$

The ratio of the number of balls in diameter of $2.5 \mathrm{~cm}$ to that of balls in diameter of $1.9 \mathrm{~cm}$ was $1 / 3$.

\begin{tabular}{llllll}
\hline & $N / N_{\mathrm{c}}$ & $k_{1} \mathrm{~s}\left(\mathrm{~cm}^{2} / \mathrm{g} \mathrm{hr}\right)$ & $k_{1} \mathrm{c}\left(\mathrm{cm}^{2} / \mathrm{g} \mathrm{hr}\right)$ & $k_{1} \mathrm{~s} / k_{1} \mathrm{c}$ & $\gamma$ \\
\hline \multirow{2}{*}{ Sulfadimethoxine } & 0.70 & $1.1 \times 10^{5}$ & $3.4 \times 10^{4}$ & 3.2 & 0.96 \\
& 1.1 & $1.2 \times 10^{5}$ & $4.2 \times 10^{4}$ & 2.9 & 0.87 \\
White alundum & 0.70 & $9.2 \times 10^{2}$ & $2.5 \times 10^{2}$ & 3.7 & 1.1 \\
& 1.1 & $7.0 \times 10^{2}$ & $2.8 \times 10^{2}$ & 2.5 & 0.75 \\
\hline
\end{tabular}

$k_{1 \mathrm{~s}}: k_{1}$ for ball-milling by stainless steel balls

$k_{1 \mathrm{e}}: k_{1}$ for ball-milling by ceramic balls

Tanaka reported that the rate of an increase of surface area by ball-milling was proportional to the product of $P_{\mathrm{c}}$ by $P_{\sigma}$, where $P_{\mathrm{c}}$ was the probability of collision between balls and powder particles and $P_{\sigma}$ was the probability for powder particles to be broken after the collision with balls. ${ }^{5)}$. Parameter $k_{1}$ is represented by equation (5) or equation (5)', when $J_{\mathrm{b}}$ is between 0.15 and 0.45 , and $N / N_{\mathrm{c}}$ is constant.

5) T. Tanaka, Ind. Eng. Chem. Process Design Develop., 5, 353 (1966). 


$$
\begin{aligned}
& k_{1}=K J_{\mathrm{b}}{ }^{a} J_{\mathrm{s}}{ }^{-\beta} \rho_{\mathrm{b}} \gamma \\
& k_{1}=K\left\{J_{\mathrm{b}}{ }^{(\alpha-\gamma)} J_{\mathrm{s}}-(\beta-\gamma)\right\}\left(\rho_{\mathrm{b}} \cdot J_{\mathrm{b}} / J_{\mathrm{s}}\right)^{\gamma}
\end{aligned}
$$

$K$ : A constant

The values of $(\alpha-\gamma),(\beta-\gamma)$ and $\gamma$ were tabullated in Table III. The energy given to a unit weight of powders in a unit time is considered to be related to the ratio of the total weight of balls to that of the sample, that is, to $\rho_{\mathrm{b}} \cdot J_{\mathrm{b}} / J_{\mathrm{s}}$. Probably, $J_{\mathrm{b}}{ }^{(\alpha-\gamma)} J_{\mathrm{s}}{ }^{-(\beta-\gamma)}$ is related to $P_{\mathrm{c}}$ and $\left(\rho_{\mathrm{b}} \cdot J_{\mathrm{b}} / J_{\mathrm{s}}\right)^{\gamma}$ is related to $P \sigma$ which is the probability concerned with crushing strength. The value of $(\beta-\gamma)$ for sulfadimethoxine is smaller than the value for white alundum. The powder particles of sulfadimethoxine are soft and sticky, while those of white alundum are hard and not so sticky as sulfadimethoxine particles. Physicochemical properties of powder particles, such as coherency between particles, may influence on $(\beta-\gamma)$.

TABLE III. The Values of $(\alpha-\gamma),(\beta-\gamma)$ and $\gamma$ for Sulfadimethoxine and White Alundum

\begin{tabular}{lcccc}
\hline \hline & $N / N_{\mathrm{C}}$ & $(\alpha-\gamma)$ & $(\beta-\gamma)$ & $\gamma$ \\
\hline Sulfadimethoxine & $0.70-0.85$ & $0.8-1.1$ & 0.4 & 1.0 \\
& 1.1 & -0.9 & 0.3 & 0.9 \\
White alundum & 0.70 & 1.0 & 0.6 & 1.1 \\
& 1.1 & -0.8 & 0.7 & 0.8 \\
\hline
\end{tabular}

